\title{
EDITORIAL
}

\section{Responsible conduct of research - Part 2}

Enoka Corea (DD https://orcid.org/0000-0003-1450-2098, Himani Molligoda (iD https://orcid.org/0000-0002-9216-9035, Co-Editors

Keywords: responsible conduct of research, scientific integrity, research integrity, research misconduct

(C) Authors. This is an open-access article distributed under a Creative Commons Attribution-Share Alike 4.0 International License (CC BY-SA 4.0), which permits unrestricted use, distribution, and reproduction in any medium, provided the original author and source are attributed and materials are shared under the same license.

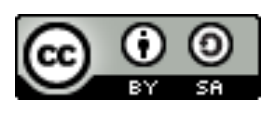

\section{Introduction}

Much of the world's progress in recent times has been due to advancements in science. For science to generate accurate results and recommendations, adherence to honest and verifiable methods in proposing, performing, evaluating, and reporting research activities is essential. How does an early career scientist do this in practice throughout the research cycle?

Responsible conduct of research begins with the research proposal. All research proposals should undergo peer review and any shortcomings corrected. Scientific validity should be ensured by powering the study adequately, including for subgroup analysis. The variable and data analysis plan should be provided in sufficient detail. The proposal should be submitted to an ethics review committee and ethics approval obtained prior to research.

Once ethics approval and scientific review has been done the researcher should ensure that he/she obtains any other permissions or authorizations. If the research is a clinical trial, it should be registered in a clinical trials registry. Other documents that may need to be finalized at this time are agreements on collaborative research and material transfer agreements. The researchers should address any conflicts of interest and mitigate them. The research should be compliant with any applicable laws, regulations, guidelines, and procedures.

After the research study is approved, it is important to use only the approved versions of the proposal, questionnaire and information sheet while obtaining required endorsements for any modifications. The researchers should set aside adequate dedicated time for conduct of the research. It is the responsibility of the researcher to ensure his/her competence and that of the research assistants. They should have the required qualifications, competence, and training to conduct the research safely and effectively.

Good research practices, including the use of standard definitions, validated methods and materials and reagents prepared using good manufacturing practices, should be followed. 
Equipment, instruments, reagents, kits, tests, questionnaires and other data collection tools, computer models etc. should be calibrated and validated. Positive and negative controls should be used as appropriate.

As resources for research are limited it is important for researcher to minimise wastage and use good accounting practices when spending grant funds. However, fair remuneration should be provided to staff. The environment should be protected with responsible use of water and energy and minimum pollution. The human and animal subjects involved in the research should be treated with respect and their welfare looked after.

Documentation is vital for quality research. Maintain a clear and accurate documentation system including records of the research methods and data sources, any approvals granted, during and after the research process and retain research data, including electronic data, in a durable, indexed, and retrievable form. Data recording should be accurate, objective, honest, transparent, and independent without fabrication or falsification. Ensure that research data and primary materials are kept in safe and secure storage and retained for at least the minimum period specified in the institutional policy. This research data should be made available for use by other researchers unless this is prevented by ethical, privacy or confidentiality matters. The statistical tests already documented in the research plan should be used correctly with sufficiently stringent criteria for statistical significance and interpreted correctly.

Publication ethics are another aspect of the responsible conduct of research. Report results using the highest standards of objectivity, transparency, and independence, without bias, exaggeration, misrepresentation, or misinterpretation. Avoid plagiarism. Co-authors should earn their place, and should have participated in the conception and design of the experiment, execution of the experiment and collection and storage of the supporting data, analysis and interpretation of the primary data or preparation and revision of the manuscript. Avoid gift authorships/ ghost authorship. However, all others who contributed to the research should be acknowledged. Once these good research practices become embedded in the psyche of the researcher they will be replicated and followed throughout the research career.

\section{References}

1. National Academy of Sciences, National Academy of Engineering, and Institute of Medicine. 1992. Responsible Science: Ensuring the Integrity of the Research Process: Volume I. Washington, DC: The National Academies Press. doi: 10.17226/1864 https://doi.org/10.17226/1864

2. Code of Conduct for Health Research in Sri Lanka (Ministry of Health) (2018) 